\title{
Mechanisms of Electroacupuncture Pretreatment in Alleviating Myocardial Ischemia Reperfusion Injury: Interactions between the Cerebellar Fastigial Nucleus and Lateral Hypothalamic Area
}

\author{
Qing Yu' ${ }^{1,2}$, Li-bin Wu' ${ }^{1}$, Fan Zhang ${ }^{1}$, Xiao-tong Wei ${ }^{1}$, Pian-pian Chen ${ }^{1}$, Shuai-ya Wang ${ }^{1}$, Mei-yi Cai ${ }^{1}$, \\ Qi Shu', Liao-yuan $\mathrm{Li}^{1}$, Zi-jian $\mathrm{Wu}^{2,3}$, Rong-lin $\mathrm{Cai}^{2,3, *}$, Ling Hu${ }^{2,3, *}$ \\ ${ }^{1}$ College of Acupuncture and Moxibustion, Anhui University of Chinese Medicine, Hefei, China \\ ${ }^{2}$ Acupuncture and Meridian Research Institute, Anhui Academy of Chinese Medicine, Hefei, China \\ ${ }^{3}$ Key Laboratory of Xin'an Medicine, Ministry of Education, Anhui University of Chinese Medicine, Hefei, China
}

Received August 26, 2021

Revised November 4, 2021

Accepted December 10, 2021

Correspondence to

Rong-lin Cai

Key Laboratory of Xin'an Medicine, Ministry of Education, Anhui University of Chinese Medicine, Hefei, China E-mail ronglincai@ahtcm.edu.cn

\section{Ling Hu}

Acupuncture and Meridian Research Institute, Anhui Academy of Chinese Medicine, Hefei, China

E-mail hulingtcm@126.com
Background: Myocardial ischemia reperfusion injury (MIRI) is an important mechanism of post-myocardial infarction injury and a main cause of death in patients with ischemic heart disease. Electroacupuncture (EA) pretreatment is effective for the prevention and treatment of MIRI, but mechanisms mediating the effects of cardiovascular disease EA treatments remain unclear.

Objectives: To determine whether the lateral hypothalamus (LHA) and the cerebellar fastigial nucleus (FN) are involved in the protective effects of EA stimulation on MIRI.

Methods: EA pretreatment was performed for 7 days before the establishment of the MIRI model. ST-segment changes on electrocardiograms were recorded and the Curtis-Walker arrhythmia score was used to evaluate changes in reperfusion injury. Hematoxylin-eosin staining was applied to evaluate the pathological and morphological changes in myocardial tissue. c-fos expression in the LHA and FN was determined by immunofluorescence staining. Glutamic (Glu) and y-Aminobutyric acid (GABA) levels were measured using a high-performance liquid chromatography-electrochemical method. Results: EA pretreatment reduced ST-segment elevation, arrhythmia scores, and morphological changes in MIRI myocardial cells in rats, and decreased the c-fos protein expression in LHA/FN nuclei. MIRI was associated with an imbalance between GABA and Glu levels, whereas EA pretreatment increased GABA levels and decreased Glu levels in the LHA/FN.

Conclusion: FN and LHA are involved in the EA-mediated attenuation of MIRI. Pretreatment with EA plays a protective role in the myocardium by regulating Glu and GABA release in the LHA and FN.

Keywords: Electroacupuncture, Lateral hypothalamic area, Cerebellar fastigial nucleus, Myocardial ischemia reperfusion injury, Heart meridian

\section{INTRODUCTION}

The aging population and lifestyle-related changes have resulted in the progressively increasing incidence of global ischemic heart disease over the years [1]. Timely restoration of vascular supply to ischemic myocardial tissue is the goal of effective treatment for ischemic heart disease [2]. However, in myocardial ischemia-reperfusion injury (MIRI) - a common complication of ischemic heart disease with very serious complication potential-myocardial damage, arrhythmia, and cardiac dysfunction occurs following vascular reperfusion. In addition to tissue and cellular metabolic dysfunction and structural alterations, brain injury is a cause of post-MIRI mortality [3,4]. MIRI is a significant public health concern. The hospital mortality rate of patients with post-myocardial infarction MIRI ranges from $6 \%$ to $14 \%$ [5] and, in 2020, MIRI-related mortality constituted $10 \%$ of the coronary heart disease mortality rate [6].

Thus, the prevention as well as safe and effective treatment of MIRI remains an important issue that the medical 
community needs to urgently address. Currently, MIRI treatment includes remote ischemic pretreatment, drug postconditioning, and percutaneous coronary intervention (PCI) [7-9], and most of these methods have some limitations. The risk of myocardial infarction increases more than $20 \%$ after PCI [10]. Inadequate treatment of ischemia-reperfusion injury results in microvascular cardiac disease, which cannot be treated by modern cardiovascular interventional medicine, and accounts for more than $90 \%$ of cardiovascular diseases [11]. Effective therapies to prevent reperfusion injury have proven elusive. Therefore, MIRI remains a major medical problem [12].

Electroacupuncture (EA) is a novel therapy that has been developed by merging traditional Chinese medicine with modern electrical technology. EA pretreatment embodies the idea of "treating before sickness" in traditional Chinese medicine. In traditional Chinese medicine, the heart meridian is closely associated with heart function, spiritual consciousness, and brain function; thus, stimulating the heart meridian can regulate heart and brain function. Shenmen (HT7) is the original acupoint of the heart meridians, whereas Tongli (HT5) is the collateral acupoint of the heart meridians. Stimulation of both Shenmen and Tongli induces good regulatory effects on cardiac function. Although the mechanisms underlying MIRI attenuation by EA pretreatment have not been fully elucidated, several clinical and experimental studies have shown that EA pretreatment can effectively prevent and treat MIRI [13-16]. We previously confirmed that acupuncture can transmit information to the brain through stimulation of the body surface, integrate information in the brain, and regulate the neuroendocrine immune system for the prevention and treatment of diseases [17-19]. Thus, acupuncture at the heart meridian point could

A

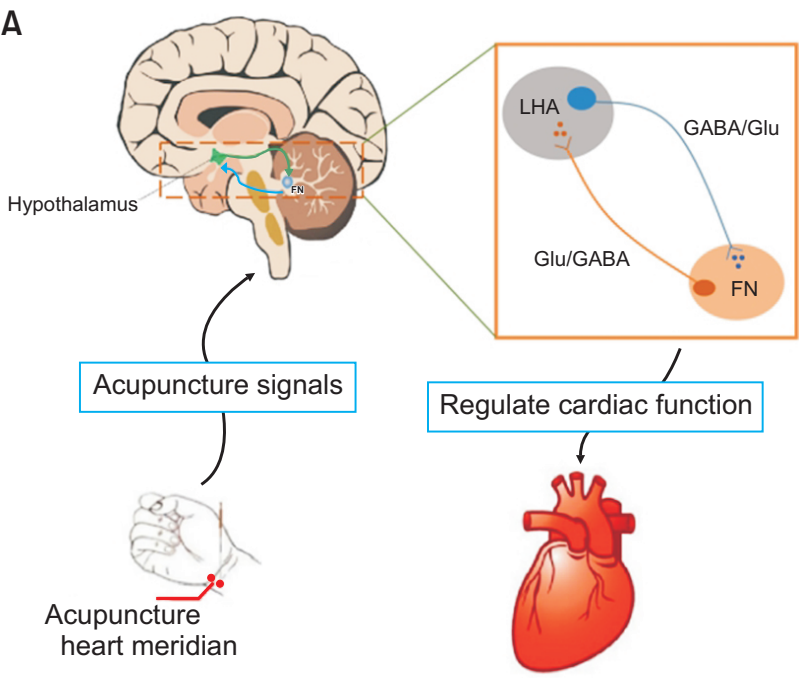

regulate the activity of neurons in the lateral hypothalamic area (LHA) and the cerebellar fastigial nucleus (FN) to alleviate MIRI [20]; however, the specific mechanisms need to be further explored.

The cerebellum is involved not only in the regulation of somatic motor function, but also in cardiovascular, digestive, and other visceral functions [21]. The hypothalamocerebellar pathway plays an important role in the integration of the somatic-visceral response [22], which provides a possible basis for defining the mechanisms mediating the integration of the somatic-visceral response. Neuroscientific studies have confirmed that the presence of a direct fiber projection between LHA and FN that comprises $\gamma$-aminobutyric acid (GABA) and glutamate (Glu) fibers [23]. The GABA and Glu levels in hypothalamic neurons are closely associated with the effects of acupuncture [24,25]. Moreover, GABA and Glu levels in the cerebellum are linked to the effects of acupuncture on blood pressure [26]; however, it is unclear whether this cerebellar effect is achieved through the LHAFN neural circuit. Furthermore, the central integration mechanism of FN, LHA, GABA, and Glu in the anti-ischemic myocardial effect of acupuncture is yet to be elucidated.

In this study, in order to further explore the connection between the cardiac meridians, heart, and nervous system, we observed the effects of acupuncture pretreatment on GABA and Glu levels in the FN and LHA of MIRI rats. Moreover, we undertook a literature review to explore the possible mechanisms that mediate the participation of the FN and LHA in the anti-acute-MIRI effect of acupuncture and the mechanism whereby acupuncture controls the hypothalamocerebellar neural circuit for regulating visceral function (Fig. 1) and compared the identified mechanisms in the context of the results.

B

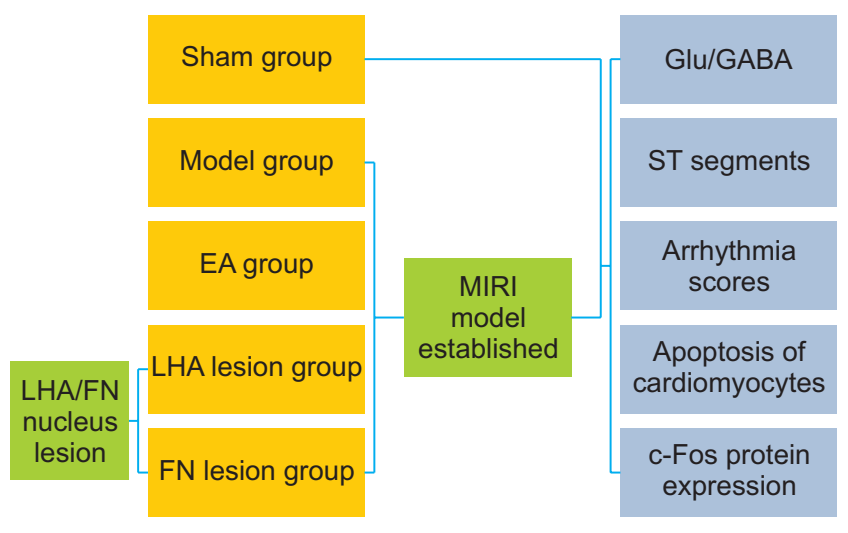

Fig. 1. Overall hypothesis (A) and study flow diagram (B). 


\section{MATERIALS AND METHODS}

In this study, we used in vivo microdialysis combined with a high-performance liquid chromatography-electrochemical detection technique (HPLC-ECD) in a rat model of MIRI.

\section{Animals and treatment groups}

Sixty male Sprague-Dawley (SD) rats (2 months old, weighing 220-250 g) without specific pathogenic infection were supplied by Anhui Medical University (SCXK (AnHui) 2017-001), and we maintained the rats in the same conditions in separate cages for 1 week. Next, the rats were numbered and randomized into five groups using a randomization table as follows: sham operation group (sham), MIRI model group (Model), acupuncture pretreatment + MIRI group (EA), FN lesion + acupuncture pretreatment + MIRI group (FN lesion), and LHA lesion + acupuncture pretreatment + MIRI group (LHA lesion). After the experimental process, the animals were euthanized in strict accordance with the guidelines for the treatment of experimental animals issued by the Ministry of Science and Technology of the People's Republic of China in 2006, with care to minimize the pain and discomfort of animals and to reduce the number of animals used.

The study protocol was approved by the Animal Ethics Committee of Anhui University of Chinese Medicine (AHUCM-rats-2020028).

\section{Reagents and instruments}

The following instruments were used: Powerlab multichannel physiological recorder (ML118, Australia Ad Instruments), automatic dehydration machine (ZT$12 \mathrm{M}$, Hubei Matt), tissue-embedding machine (YB7LF, Hubei Matt), Leica slicer (RM2016, German Leica), fluorescence microscope (CX41,OLYMPUS), rat brain stereotaxis (Sweden CMA), microdialysis pump (CMA/400, Sweden CMA), Milli-Q Ultra-Pure Water system (IQ7000, Millipore USA), electrochemical detector (DECADEII, Holland ANTEC), cryogenic microcollector (EFC-82, Japan EICOM), cryogenic regulator (EFR-82, Japan EICOM), brain stereotaxic skull drill (78001, Reward), Microdialysis probe (membrane length $4 \mathrm{~mm}$, Reward), probe catheter (RWD/12, Reward), microinjector (CMA1mL, CMA, Sweden), and electroacupuncture instrument (SDZ-V, Suzhou Medical Supplies Factory Co., Ltd.)

The following reagents were used: artificial cerebrospinal fluid, disodium ethylenediamine tetraacetate, kainic acid, ascorbic acid, anhydrous acetic acid (20180812), and serotonin hydrochloride (BO1022DA14; from Shanghai Yuanye Biotechnology Co., Ltd.), rabbit source c-fos first antibody (208942, UK Abcam), $\gamma$-aminobutyric acid standard (A21528, American Sigma), glutamic acid standard (V1001, American
Sigma), chloral hydrate (201805 National Pharmaceutical Group Chemical Reagent Co., Ltd.), imported sheep serum working solution (WK173310, Beijing Zsbio), fluorescent second antibody (GR3203087-1, UK Abcam), DAPI staining solution (071618190213, Shanghai Beyotime), antifluorescence quenching sealing solution (111318190306, Shanghai Beyotime), hematoxylin dye solution (20200725, Zhuhai Baso), and eosin dye solution (20200727, Zhuhai Baso).

\section{Interventional procedure}

Before the MIRI experiment, rats in the EA group were pretreated with EA (for 7 days) whereas rats in the LHA and FN lesion groups received acupuncture intervention 3 days after the LHA/FN nucleus lesion. Based on a previous study [27], the "Shenmen" (HT7) and "Tongli" (HT5) sections of Shaoyin Heart Meridian of the hand were selected as the acupoint positioning standard for experimental acupuncture and moxibustion treatment in rats. The acupuncture points were selected from HT7, located along the ulnar margin with transverse striation on the medial wrist of the forelimb, and HT5, located in the medial forelimb, $1 \mathrm{~mm}$ above the transverse carpal striation. To minimize the stress induced by animal restraint, the rats received preprocedural light anesthesia with isoflurane in oxygen flow. Sterile acupuncture needles (diameter, $0.3 \mathrm{~mm}$; length, $25 \mathrm{~mm}$ ) were inserted into the Shenmen (HT7)-Tongli (HT5) points to a depth of $2 \mathrm{~mm}$. Bilateral Shenmen (HT7)-Tongli (HT5) points were connected to the EA treatment instrument (SDZ-V; continuous wave, frequency $2 \mathrm{~Hz}$, intensity $1 \mathrm{~mA}$, duration $\geq 20$ minutes per day for 7 days). MIRI was induced on the eighth day.

\section{Animal model}

In this study, the MIRI model was established in rats by the physical ligation and push tube method [28]. Rats were anesthetized with sodium pentobarbital $(100 \mathrm{mg} /$ $\mathrm{kg}$ intraperitoneal) $24 \mathrm{~h}$ after the final EA and fixed in the supine position. A standard II lead electrocardiogram (ECG) was recorded in real time using the Powerlab physiological recorder. The skin between the left third and fourth intercostal spaces of the rat was cut, the muscle was bluntly separated, and the pleura was bluntly separated with hemostatic forceps and opened. The pericardial capsule was cut to expose the heart, and a $2 \times 6$ round needle with a No. 6-0 medical nylon monofilament thread at 2 to 3 $\mathrm{mm}$ below the left atrial appendage was used to bypass the anterior descending coronary artery and was drawn out from the paracone groove of the pulmonary artery. A pressure equalizer polyethylene (PE) tube was placed on the silk thread and a slip knot was used to establish an ischemia model. The left ventricular anterior wall swelled outwards, and the 
elevation of the ST-segment and T-wave inversion on the ECG were used to confirm myocardial ischemia. The left anterior descending artery (LAD) was ligated for 30 minutes, followed by 120 minutes of reperfusion. During reperfusion, the elevated ST segment decreased by more than half, indicating that the I/R model was established successfully. Rats in the model, EA, FN lesion, and LHA lesion groups underwent MIRI surgery. In the sham group, there was no ligation after thoracotomy and the needle was inserted only once at the corresponding site.

\section{Nucleus lesioning}

In this experiment, a nuclear lesion of the rat was induced by the chemical method. In the FN and LHA lesion groups, kainic acid was injected into the bilateral FN and LHA, respectively. Rats were anesthetized with an intraperitoneal injection of pentobarbital sodium $(100 \mathrm{mg} / \mathrm{kg})$ and fixed on the brain stereotaxic instrument and the nucleus coordinates of the FN or LHA were established (FN: Bregma: $-11.6 \mathrm{~mm}$, LR: $1.0 \mathrm{~mm}$, and H: $5.6 \mathrm{~mm}$, LHA: Bregma: $-2.8 \mathrm{~mm}$, LR: 1.5 $\mathrm{mm}$, and H: $8.3-8.5 \mathrm{~mm}$ ), in accordance with the rat brain atlas [29]. After determining the location of the nucleus, a hole was drilled into the skull, a microinjector was slowly inserted into the predetermined site, and $0.4 \mu \mathrm{L}$ kainic acid (dose: $1 \mathrm{~g} / \mathrm{L}$ ) was slowly injected into each side of the relevant nucleus (Fig. 2). The needle was retained for 5 minutes post injection, and was then slowly removed before suturing the wound. Three days after surgery, significant neuronal cell death was observed in the FN and LHA regions.

\section{Cardiovascular assessment}

The ECG of the rats was recorded using the Power Lab 8/30 recorder systems. The displacement of the ST segment for 15 min was analyzed at each time point. The Curtis-Walker arrhythmia score method [30] was used to evaluate the score after reperfusion in each group (score 0 -4: normal $=0$, atrial arrhythmia or occasional ventricular extrasystole $=1$, frequent ventricular extrasystole $=2$, ventricular tachycardia
[1-2 series] $=3$, ventricular tachycardia [ $>3]$, or ventricular fibrillation $=4$ ).

\section{Detection of pathological changes of myocardial tissue by Hemolysin-Eosin (HE) staining}

HE staining was applied to assess the pathological and morphological changes in the myocardial tissue. Myocardial tissues were fixed in $4 \%$ paraformaldehyde for 24 hours, and $3-\mu \mathrm{m}$ slices were dewaxed and stained with HE. Morphological changes of the myocardium were observed under light microscopy.

\section{Immunofluorescence analysis}

The expression of c-fos in LHA/FN was examined by immunofluorescence staining. After $120 \mathrm{~min}$ of reperfusion, rats were anesthetized with sodium pentobarbital $(100 \mathrm{mg} /$ $\mathrm{kg}$ administered intraperitoneally). The heart of the rats was transcardially perfused with $0.9 \%$ saline followed by $4 \%$ paraformaldehyde, and the brain was harvested and fixed by perfusion with $4 \%$ paraformaldehyde for 24 hours before being embedded in paraffin. After dehydration, embedding, and slicing, six sections of each sample containing the target area of the brain were selected for staining. Tissues were fixed for 30 minutes, washed thrice with PBS, and blocked for 30 minutes with $10 \%$ goat serum. After the serum was removed, the primary antibody anti-c-fos (1:500, goat; Santa Cruz) was added and incubated for 1 hour followed by washing three times with $\mathrm{PBS}$ at $37^{\circ} \mathrm{C}$. A fluorescencelabeled secondary antibody (Abcam, ab150077, GR32030871) was added to the brain slices and the section was then stained with DAPI (Beyotime, C1005, 071618190213). Finally, an anti-fluorescence quencher was used to seal the sections. Fluorescence images of target brain areas were scanned and photographed using the OLYMPUS automatic virtual slice scanning system (VE120). c-Fos-positive cells were counted using ImageJ. The mean number of c-fos positive cells per LHA and FN nuclei for each animal was determined by averaging the total counts from different rats in each group.
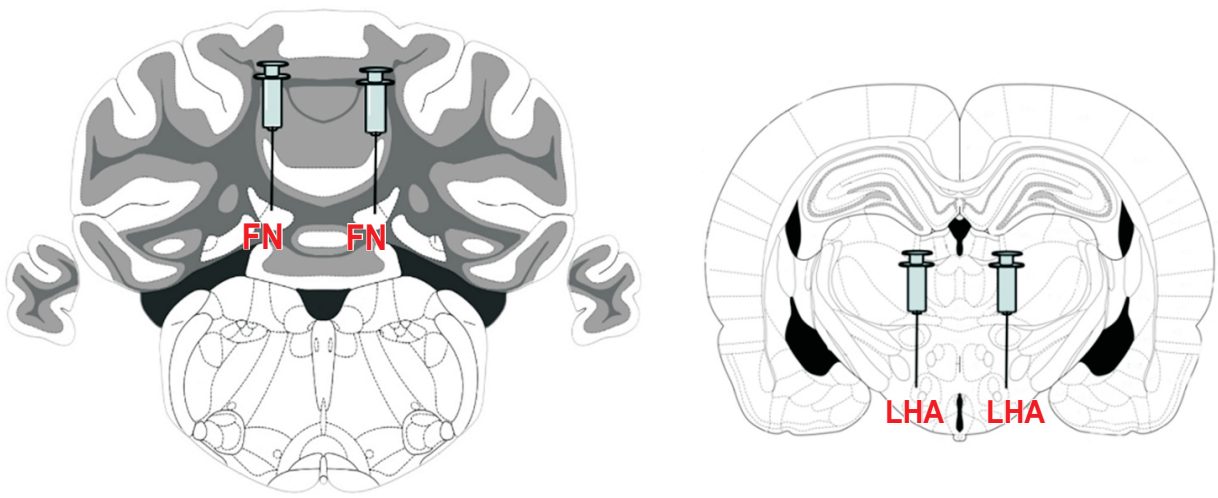

Fig. 2. A schematic for KA injection sites. $\mathrm{FN}=$ fastigial nucleus; $\mathrm{LHA}=$ lateral hypothalamus. 


\section{Microdialysis}

Using the EICOM microdialysis system, the microdialysis probe was inserted into the corresponding nucleus and fixed. Six rats each in the sham, model, and EA groups were selected for the collection of brain microdialysate of LHA and FN. In the FN lesion and LHA lesion groups, LHA and FN brain microdialysates, respectively, were collected. Artificial cerebrospinal fluid was extracted using a 1-mL microinjector, one end of the probe was connected to the perfusion system, and the other end was placed in a cryogenic microcollector to collect the dialysate. The online microdialysis system continuously infused $10 \mu \mathrm{mol} / \mathrm{L}$ artificial cerebrospinal fluid at a $2.0 \mu \mathrm{L} /$ minute flowrate. A tube of dialysate was collected every 15 minutes and the intercellular fluid from each area of the brain was microsampled. GABA and Glu levels in LHA and FN dialysates were quantified by the HPLC-ECD system.

\section{Statistical analysis}

All statistical analysis were performed using GraphPad Prism version 8 (GraphPad Software Inc., USA), and values are expressed as mean \pm standard deviation $($ mean $\pm \mathrm{SD})$. When the data were normally distributed and the variance was uniform, a single-factor analysis of variance was used and the LSD method was used for intergroup comparison. When the measurement data did not conform to a normal distribution or the variance was non-uniform, a nonparametric test was used. A statistically significant difference was established with $p<0.05$.

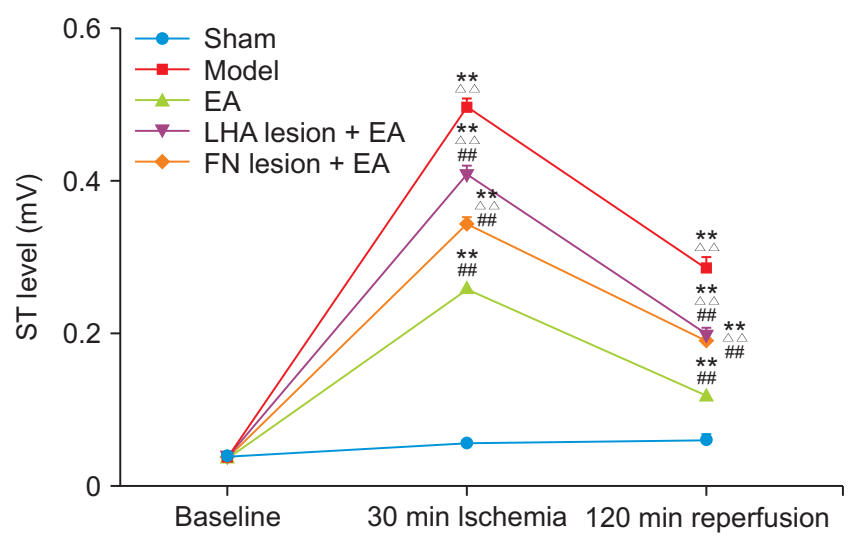

Fig. 3. The change of ST segments in different groups. Data were expressed as mean $\pm \mathrm{SD},{ }^{*} p<0.05,{ }^{* *} p<$ 0.01 , compared with sham group, ${ }^{*} p<0.05,{ }^{* \#} p<0.01$ compared with model group, $\Delta p<0.05, \Delta \Delta p<0.01$, compared with EA group. $\mathrm{n}=12$; Sham $=$ control group; Model = myocardial ischemia reperfusion group; $\mathrm{EA}=$ electroacupuncture treatment group; LHA lesion $+E A=$ electroacupuncture (EA) in LHA lesioned group; FN lesion + $\mathrm{EA}=$ electroacupuncture (EA) in FN lesioned group.

\section{RESULTS}

\section{Acupuncture pretreatment attenuated electrocardiogram ST-segment displacement}

There were no statistically significant differences in the ST segment between the sham, model, EA, FN lesion, and LHA lesion groups before ligation ( $p>0.05$, respectively; Fig. 3). The post-ligation and reperfusion ST segments in the model, EA, FN lesion, and LHA lesion groups were significantly higher than in the sham group ( $p<0.05$, respectively), suggesting that myocardial ischemia and reperfusion caused abnormal elevation of the ST segment. At 30 minutes of ligation and 120 minutes of reperfusion, the elevation of the ST segment in the EA group was significantly lower than that in the model group, indicating that EA pretreatment can significantly inhibit the MIRI-induced abnormal elevation of the ST segment. Interestingly, the ST-segment elevation was higher in the LHA/FN lesion group than in the EA group 30 minutes after ligation and 120 minutes after reperfusion, indicating that the inhibitory effect of EA pretreatment on the elevated ST segment was affected by the FN/LHA nucleus.

\section{Acupuncture pretreatment attenuated arrhythmia scores}

Compared to the sham group, the arrhythmia score was significantly increased in the model group $(p<0.01)$; however, the arrhythmia score in the EA group was significantly lower than that of the model group $(p<0.01)$. The arrhythmia score of the LHA/FN lesion group was higher than that of the EA group $(p<0.01)$ (Fig. 4).

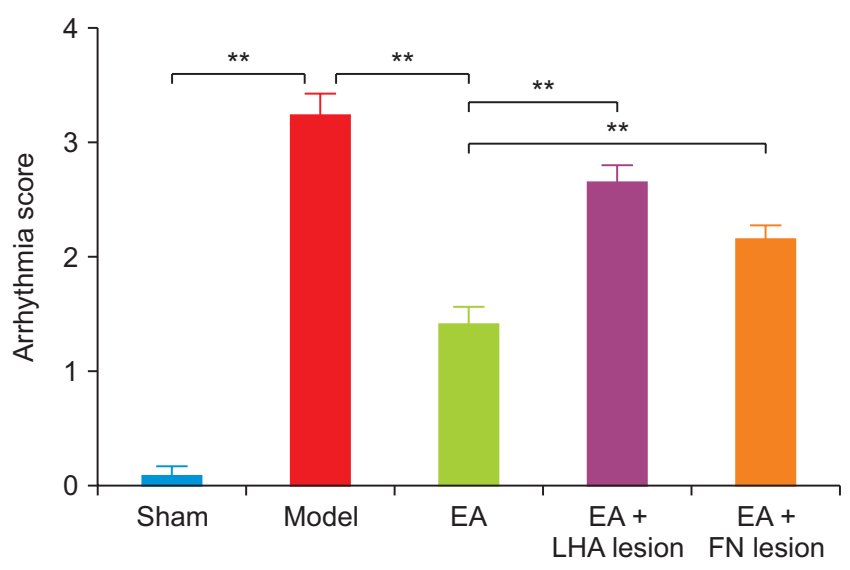

Fig. 4. Comparison of arrhythmia scores between rats in different groups. Data are expressed as mean $\pm S D$, ${ }^{*} p<0.05,{ }^{* *} p<0.01, \mathrm{n}=12$. Sham = control group; Model = myocardial ischemia reperfusion group; $\mathrm{EA}=$ electroacupuncture treatment group; LHA lesion + EA = electroacupuncture (EA) in LHA lesioned group; FN lesion + $\mathrm{EA}=$ electroacupuncture $(\mathrm{EA})$ in $\mathrm{FN}$ lesioned group. 

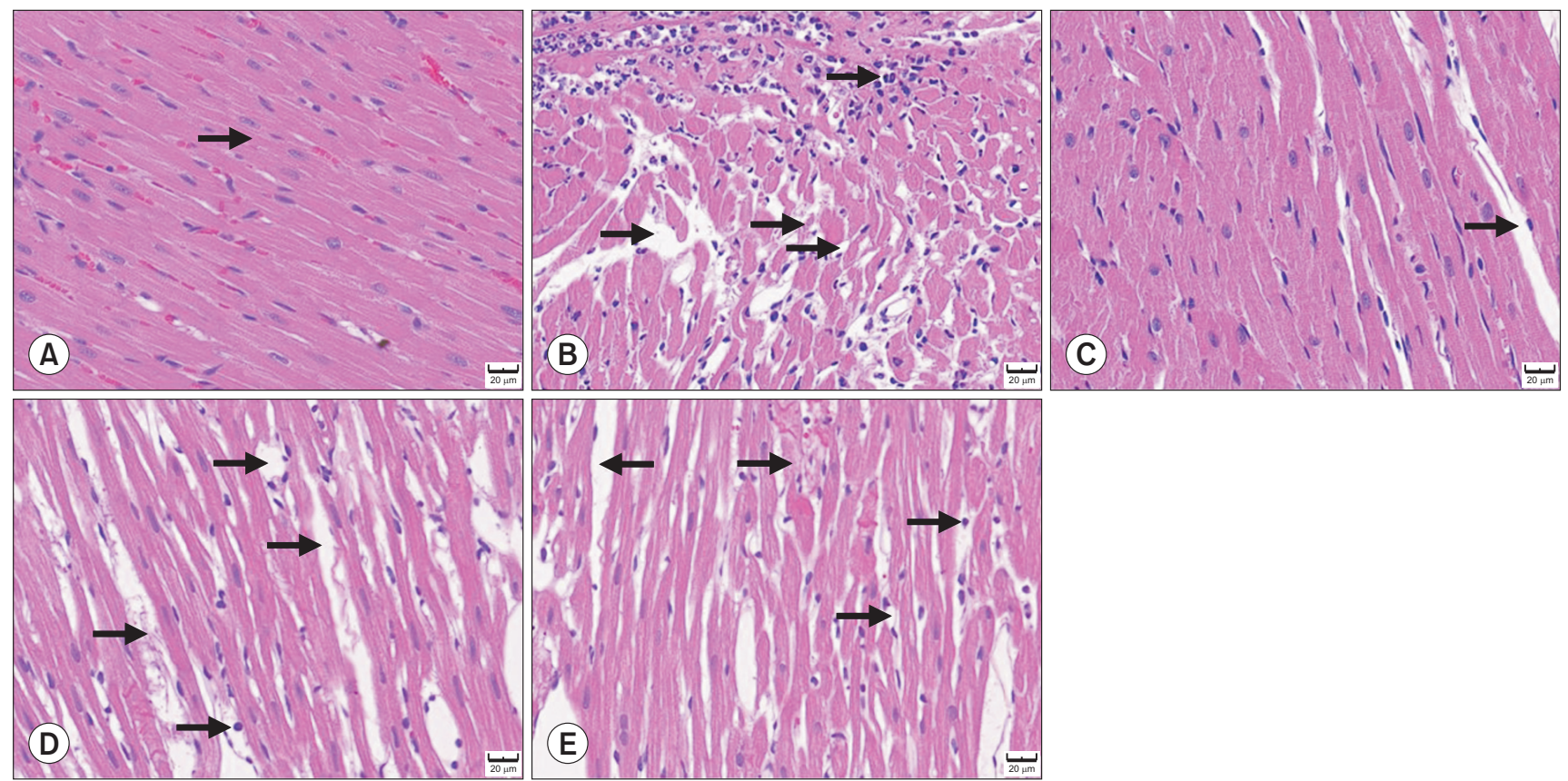

Fig. 5. Myocardial tissue of rats in different group (HE staining, 400x, scale $20 \mu \mathrm{m}$ ), representative images for sham group (A), model group (B), EA group (C), LHA lesion + EA group (D), FN lesion + EA group (E). Sham = control group; Model = myocardial ischemia reperfusion group; $E A=$ electroacupuncture treatment group; LHA lesion $+E A=$ electroacupuncture (EA) in LHA lesioned group; FN lesion + EA = electroacupuncture (EA) in FN lesioned group.
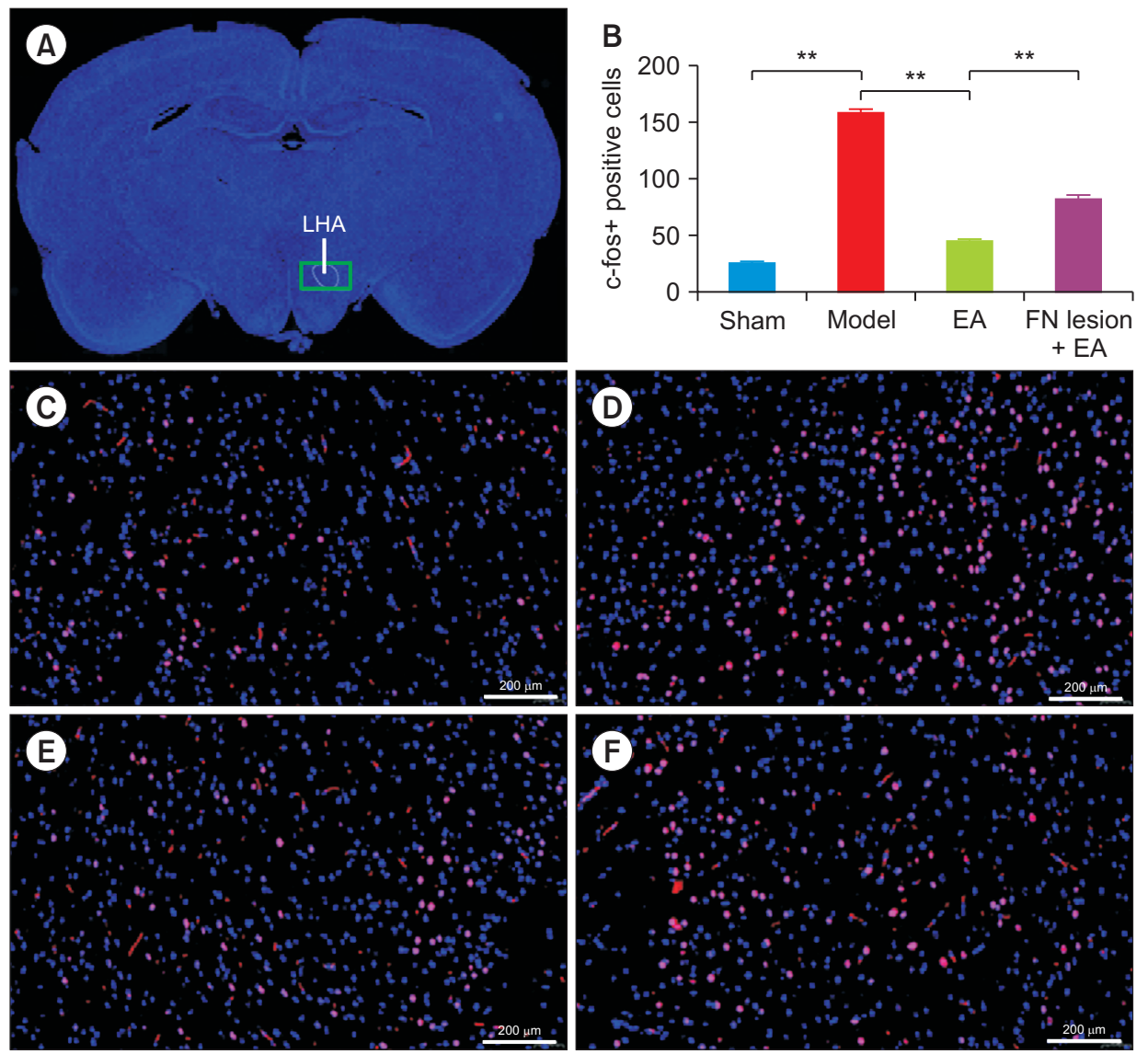

Fig. 6. The c-fos protein expression in LHA of rats in different groups. Red represents c-fos positive cells and blue represents DAPI immunostaining. Localization of LHA brain region in rats $(A)$, quantification data for the $\mathrm{c}$-fos positive cell numbers (B) and representative images for sham group (C), model group (D), EA group (E) and FN lesion + EA group (F). Data were expressed as mean $\pm \mathrm{SD}$. ${ }^{*} p<0.05,{ }^{* *} p<0.01, \mathrm{n}=3$, Sham = control group; Model = myocardial ischemia reperfusion group; $\mathrm{EA}=$ electroacupuncture treatment group; $\mathrm{FN}$ lesion + EA = electroacupuncture (EA) in FN lesioned group. 


\section{Acupuncture pretreatment alleviated apoptosis of cardiomyocytes}

Normal morphology with tight and well-ordered arrangement of cardiomyocytes without necrosis of muscle fibers was observed in the sham group. The model group showed enlargement of the myocardial fiber space, leukocyte infiltration, rupture of myocardial fibers, interstitial edema, cardiomyocyte proliferation, and dissolved and disordered myocardial fibers. In the EA group, a small amount of the fibers of myocardial tissue were loosely and irregularly arranged, indicating that EA pretreatment alleviated apoptosis of rat cardiomyocytes. Cardiomyocyte and muscle fiber damage was moderately more serious in the LHA/FN lesion group than in the EA group (Fig. 5).

\section{Acupuncture pretreatment attenuated c-fos protein expression in the brain FN and LHA regions}

The c-fos protein expression in the LHA region was significantly higher in the model group than in other groups $(p<0.01$; Fig. 6). The $c$-fos protein expression in the EA group was significantly lower than in the model group $(p<0.01)$ and was significantly higher than in the FN lesion group $(p<0.01)$. These results suggested that FN was involved in acupuncture pretreatment for ameliorating MIRI. Similarly, $\mathrm{c}$-fos protein expression in the FN region of the model group was significantly higher than in other groups $(p<0.01$; Fig. 7$)$. The $c$-fos protein expression in of EA group was significantly lower than in the model group $(p<0.01)$, but was significantly higher than that of the LHA lesion group $(p<0.01)$. The findings suggested that LHA was involved in acupuncture pretreatment for ameliorating MIRI and that the FN and LHA interact and participate in the regulation of acupuncture pretreatment to improve cardiac function.

\section{Glu and GABA in LHA/FN participate in EA pretreatment to attenuate MIRI}

The Glu level in the LHA was significantly higher, whereas the GABA level in the model group was significantly lower than that of other groups $(p<0.01)$. The Glu level was lower, whereas the GABA level in the EA group was significantly higher than in the FN lesion group ( $p<0.01$; Fig. 8 ). In the FN of the model group, the Glu level was significantly higher whereas the GABA level was significantly lower than that of
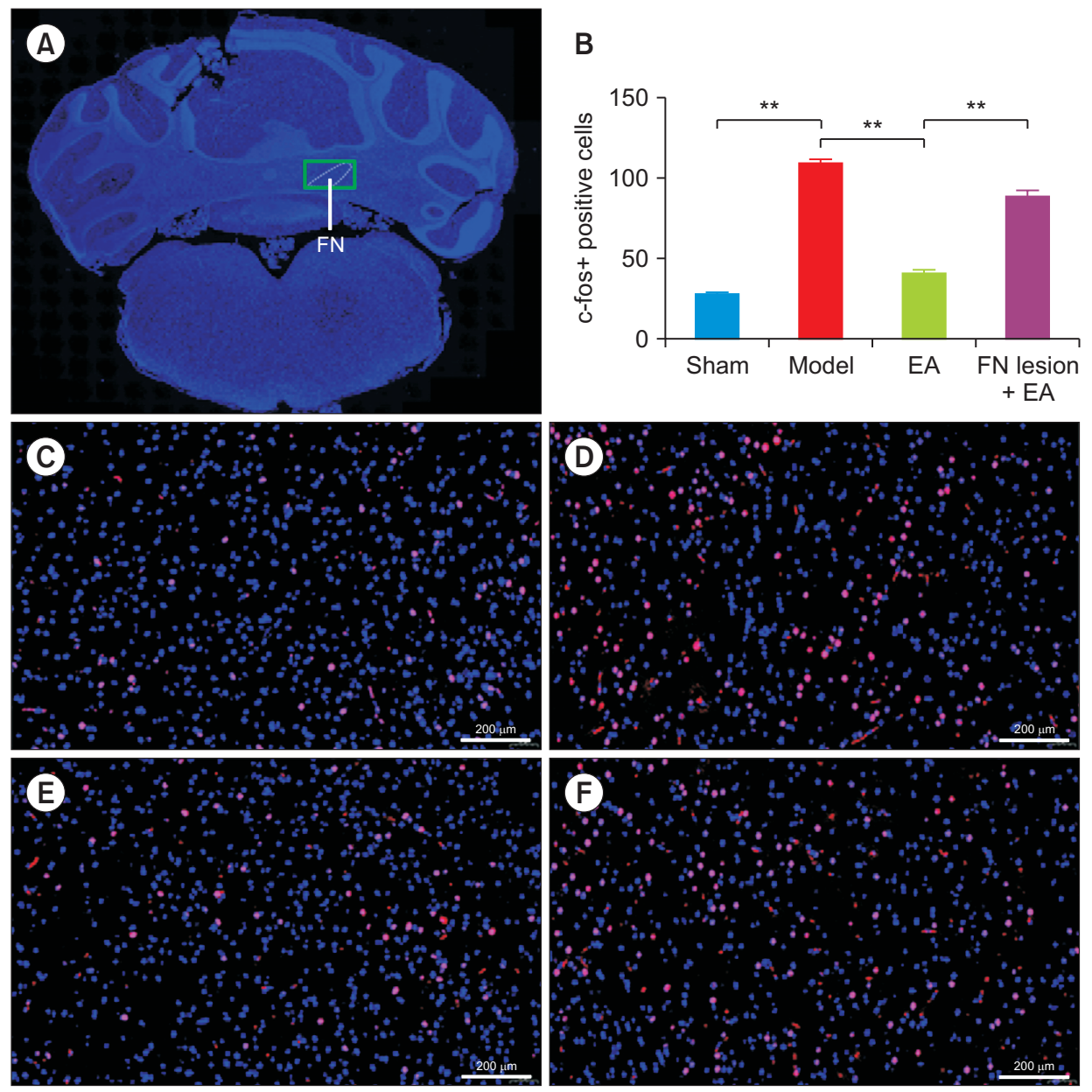

Fig. 7. The c-fos protein expression in $\mathrm{FN}$ of rats in different groups. Red represents c-fos positive cells and blue represents DAPI immunostaining. Localization of $\mathrm{FN}$ brain region in rats (A), quantification data for the C-fos positive cell numbers (B) and representative images for sham group $(\mathrm{C})$, model group (D), EA group (E) and LHA lesion + EA group $(\mathrm{F})$. Data were expressed as mean $\pm \mathrm{SD},{ }^{*} p<$ 0.05. ${ }^{* *} p<0.01 . \mathrm{n}=3$; Sham $=$ control group; Model = myocardial ischemia reperfusion group; $\mathrm{EA}=$ electroacupuncture treatment group; LHA lesion + $\mathrm{EA}=$ electroacupuncture $(\mathrm{EA})$ in LHA lesioned group. 

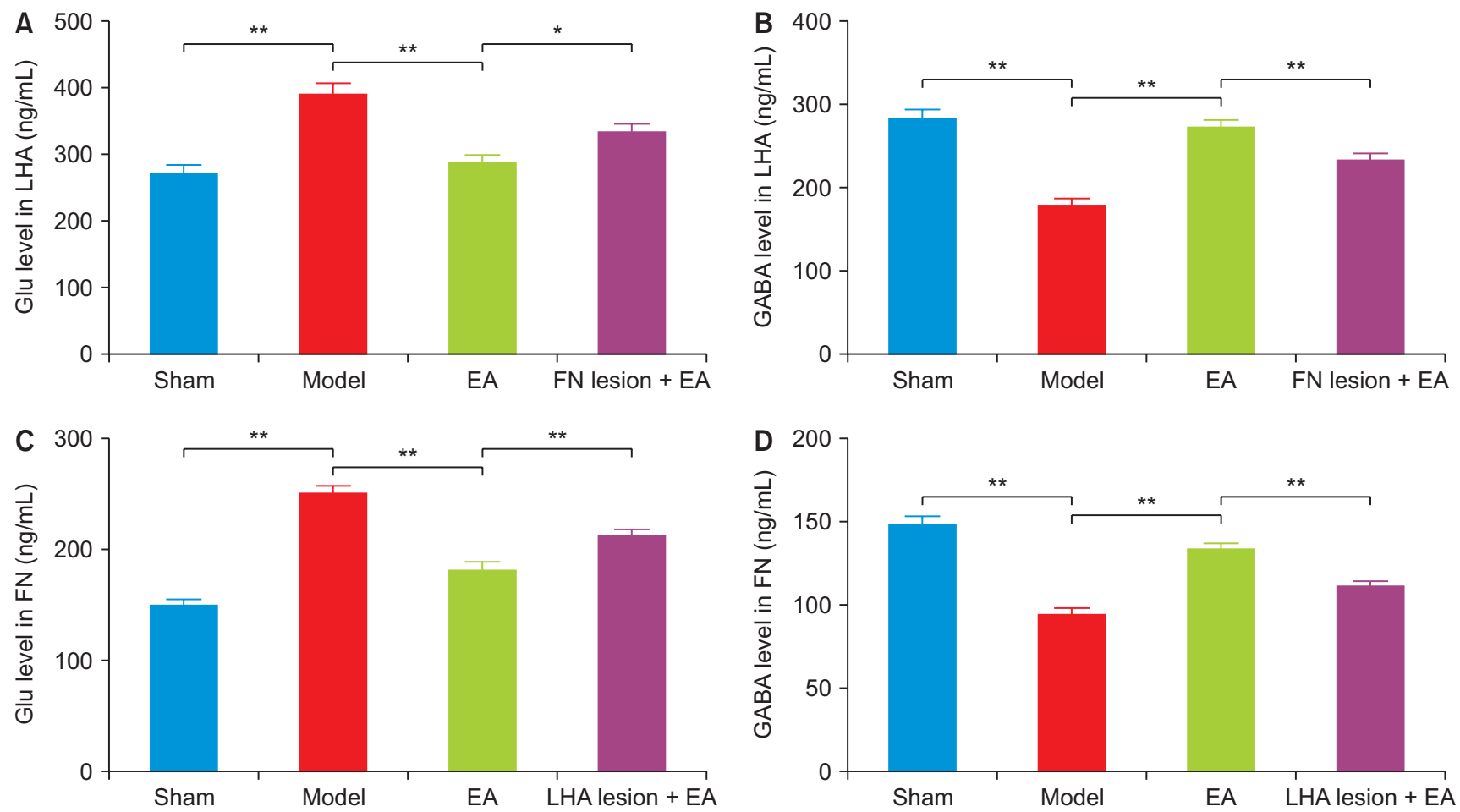

Fig. 8. The content of Glu and GABA in LHA (A, B)/FN (C, D). Data were expressed as mean \pm SD. ${ }^{*} p<0.05, * * p<0.01$. $\mathrm{n}=6$; Sham = contro I group; Model = myocardial ischemia reperfusion group; $\mathrm{EA}=$ electroacupuncture treatment group; $\mathrm{FN}$ lesion $+\mathrm{EA}=$ electroacupuncture (EA) in FN lesioned group; LHA lesion + EA = electroacupuncture (EA) in LHA lesioned group.

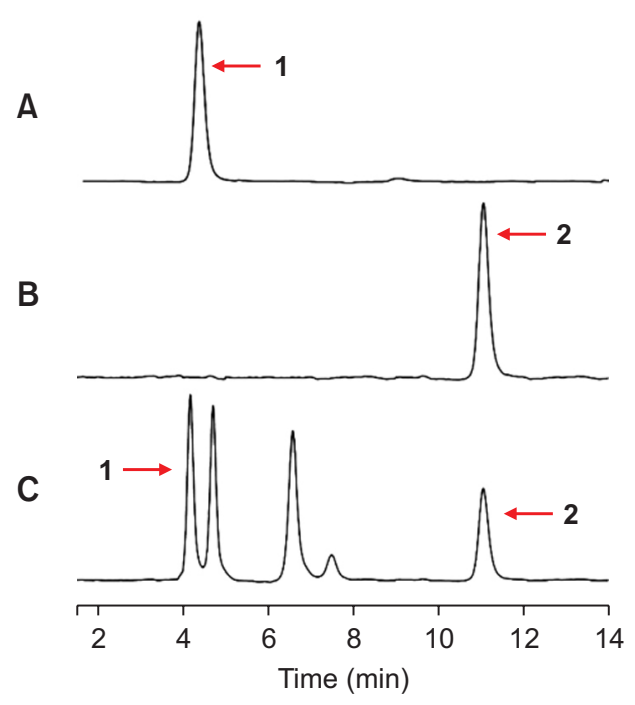

Fig. 9. HPLC chromatogram of Glu and GABA. Representative images for standards (A, B) and dialysate $(\mathrm{C}) .1$ and 2 indicate Glu and GABA, respectively.

other groups $(p<0.01)$. In the EA group, the Glu level was lower whereas the GABA level was significantly higher than that of the LHA lesion group ( $p<0.01$; Fig. 8). The HPLC chromatogram of Glu and GABA is shown in Fig. 9.

\section{DISCUSSION}

MIRI is caused by restoration of the blood supply after myocardial ischemia. Prevention of MIRI is of great significance in the treatment of acute myocardial infarction. MIRI is common in patients with cardiovascular disease and in patients undergoing cardiopulmonary bypass and heart surgery [31]. Currently, pretreatment is often used in the treatment of MIRI and involves short-term non-destructive stimulation before injury onset in order to improve the tolerance of tissues or organs to subsequent permanent and fatal injury, and is considered one of the most effective mechanisms of endogenous protection [32]. EA pretreatment can attenuate MIRI and improve the quality of life of patients [13-16]. Basic research and clinical experiments indicate that the biochemical, morphological, and genetic indices of cardiomyocytes are altered following EA pretreatment, which can activate endogenous self-protective mechanisms and resistance against disease damage that may occur in later stages [33]. Our results showed that EA pretreatment reduced the elevation of the ST segment in the ECG, arrhythmia scores, and alteration of the morphology of myocardial cells of MIRI rats, similar to the findings of previous studies [34-37]. However, the specific mechanism remains to be elucidated. 
The hypothalamus is an important center coordinating visceral responses, with LHA and other nuclei involved in the regulation of cardiovascular activity [38]. FN is also an important structure affecting cardiovascular, respiratory, and gastrointestinal activity [39]. Several studies have shown that FN participates in preventive and therapeutic neurogenic mechanisms to protect the heart, brain, and other important organs [40]. Electrical stimulation of the rostral cerebellar FN has a protective effect in surgically induced myocardial infarction in rats, and this may be due to increased vagal and decreased sympathetic tone [41]. FN and LHA are closely related to cardiovascular function. Our findings showed that, in the MIRI group, the expression of nuclear c-fos protein in the LHA and FN was significantly higher than in other groups, and decreased markedly following EA pretreatment. Meanwhile, the expression of c-fos decreased in the LHA/ FN lesion group, suggesting that the central mechanisms of EA pretreatment in attenuating MIRI was related to the interaction between LHA and FN regions.

Neuroscientific research has shown that there are direct projections of GABA and Glu fibers between FN and LHA. Glu is an excitatory neurotransmitter that is present in high levels in brain tissue, whereas GABA is an inhibitory neurotransmitter. Some studies have shown that Glu and GABA in LHA may derive from axonal endings or glial cells of the FN. High-frequency electrical stimulation of FN can inhibit the electrical activity of LHA neurons and increases the levels of neurotransmitters in the stimulated area. The inhibitory effect of high frequency electrical stimulation on LHA neurons can be achieved through direct fiber projections of FN-LHA [42-44]. Moreover, the effect of acupuncture is closely related to the regulation of neurotransmitters such as GABA and Glu in specific brain regions $[45,46]$, which may be an important means for acupuncture to regulate body function and treat diseases.

Our results showed that the ST-segment displacement and the arrhythmia score increased significantly, Glu levels in the LHA and FN increased significantly, and GABA levels in the LHA and FN markedly decreased in MIRI rats. The myocardial fiber space was enlarged, cardiomyocytes proliferated, leukocyte infiltration increased as did rupture of myocardial fibers, and interstitial edema, and the myocardial fibers were absent or disordered in MIRI rats. These findings indicated that the myocardial blood supply in MIRI rats was insufficient, the release of amino acid neurotransmitter GABA in LHA and FN decreased significantly, and the release of Glu increased significantly. Overall, MIRI was associated with an imbalance between GABA and Glu levels.

The ST-segment displacement and arrhythmia score of MIRI rats decreased significantly, the content of GABA in FN and LHA increased significantly, and the content of
Glu decreased significantly after EA pretreatment at heart meridian points. Furthermore, compared to the EA group, the GABA content in the FN of the LHA lesion group and the LHA of the FN lesion group was lower than that of the EA group, and the Glu levels were higher than in the EA group. These changes were significantly reversed by EA pretreatment at the heart meridian acupuncture points, suggesting that acupuncture may play a protective role in the myocardium by regulating the release of GABA and Glu in FN and LHA. LHA/FN play an important role in improving MIRI of EA pretreatment. These results provided an important basis for exploring the cerebellar hypothalamic pathway involved in the effects of acupuncture.

The abovementioned findings showed that EA pretreatment can not only reduce MIRI, but also promotes the release of GABA in FN and LHA of MIRI rats and inhibits the release of Glu. After damaging FN or LHA, the LHA-FN neural connection was impaired, and the regulatory effect of EA was significantly reduced. These results suggest that the amino acid neurotransmitters GABA and Glu are closely related to the protective effects of the heart meridian EA on myocardial ischemia. Furthermore, the LHA-FN neural circuit participates in the effects of acupuncture pretreatment on alleviating myocardial injury by regulating the release of GABA and Glu in LHA-FN.

The hypothalamic sympathetic lymphocyte pathway of FN GABAergic or glutaminergic neurons mediates the regulation of FN immune system function $[42,47]$. There are direct GABAergic and glutaminergic fiber projections between FN and LHA. Therefore, it is possible to that the LHA-FN GABAergic/glutaminergic nerve circuit is the key route for cerebellar participation in the acupuncture pretreatment effects to alleviate MIRI. Acupuncture pretreatment may protect the myocardium by regulating GABA and Glu levels in the LHA-FN nerve circuit and through the hypothalamicsympathetic nerve pathway.

However, this study focused only on the role of the hypothalamus and FN in the mechanism of acupuncture. Other regions and brain nuclei may also be involved in the effects of acupuncture. For example, studies on the cerebellum and brainstem have shown that they are closely related to cardiac function. At the same time, the cerebellum and brainstem are closely connected to upstream pathways in neuroanatomy. Therefore, the nuclei of brainstem and their connection with cerebellum play a role in the mechanisms induced by acupuncture to regulate cardiac function. During myocardial infarction, the cardiac sympathetic nerve is activated, the heart is overloaded, the myocardial contractility is significantly reduced, and the cardiac pump function is impaired [48]. The effect of acupuncture on the peripheral nervous system through the central nervous system is worth 
elucidating.

Future research should include additional neurobiological techniques, such as virus tracer or photogenetic, chemical, or genetic techniques to better understand the regulation of neural circuit activity by specific neurons in related brain regions, and to provide new evidence clarifying the mechanisms of acupuncture effects from the perspective of neural circuit regulation.

\section{CONCLUSIONS}

FN and LHA are involved in the mechanisms whereby EA pretreatment at the heart meridian attenuates MIRI. EA pretreatment confers myocardial protection by regulating the release of Glu and GABA in the LHA and FN.

\section{FUNDING}

This study was supported by grants from the National Natural Science Foundation of China (grant nos. 81774414, 8197375, 82074536, and 82104999), the Project for the Cultivation of Outstanding and Top Talents in Universities of Anhui Province (grant no. gxgwfx2019025), and the Nature Science Research Project of Anhui Province (grant nos. 2108085Y30 and 2108085QH364).

\section{AUTHORS' CONTRIBUTIONS}

Ling $\mathrm{Hu}$ and Rong-lin Cai conceived and supervised the study; Qing Yu and Rong-lin Cai designed the experiments; Qing Yu, Fan Zhang, Xiao-tong Wei, Pian-pian Chen, Shuaiya Wang, Qi Shu, and Mei-yi Cai performed the experiments; Zi-jian Wu and Li-bin Wu analyzed data; and Qing Yu wrote the manuscript. All authors reviewed the results and approved the final version of the manuscript.

\section{DATA AVAILABILITY}

The original data supporting the findings of this study are available from the corresponding author upon reasonable request.

\section{CONFLICT OF INTEREST}

The authors declare no conflict of interest.

\section{ORCID}

Qing $\mathrm{Yu}$,

Li-bin $\mathrm{Wu}$,

Fan Zhang,
Xiao-tong Wei,

Pian-pian Chen,

Shuai-ya Wang,

Mei-yi Cai,

Qi Shu, Liao-yuan Li, Zi-jian $\mathrm{Wu}$, Rong-lin Cai, Ling $\mathrm{Hu}$, https://orcid.org/0000-0001-7734-4299 https://orcid.org/0000-0002-2987-7012 https://orcid.org/0000-0001-6553-0537 https://orcid.org/0000-0002-9016-6922 https://orcid.org/0000-0002-7322-0798 https://orcid.org/0000-0003-0260-9357 https://orcid.org/0000-0002-6291-5404 https://orcid.org/0000-0001-5666-6280 https://orcid.org/0000-0002-8317-5433

\section{REFERENCES}

1. Virani SS, Alonso A, Benjamin EJ, Bittencourt MS, Callaway CW, Carson AP, et al. Heart disease and stroke statistics-2020 update: a report from the American Heart Association. Circulation 2020;141:e139-596.

2. Kishore SP, Blank E, Heller DJ, Patel A, Peters A, Price M, et al. Modernizing the World Health Organization list of essential medicines for preventing and controlling cardiovascular diseases. J Am Coll Cardiol 2018;71:564-74.

3. Pradita-Ukrit S, Vattanavanit V. Efficacy of thiamine in the treatment of postcardiac arrest patients: a randomized controlled study. Crit Care Res Pract 2020;2020:2981079.

4. Magruder JT, Crawford TC, Lin YA, Zhang F, Grimm JC, Kannan RM, et al. Selective localization of a novel dendrimer nanoparticle in myocardial ischemia-reperfusion injury. Ann Thorac Surg 2017;104:891-8.

5. Keeley EC, Boura JA, Grines CL. Primary angioplasty versus intravenous thrombolytic therapy for acute myocardial infarction: a quantitative review of 23 randomised trials. Lancet 2003; 361:13-20.

6. Hausenloy DJ, Yellon DM. Myocardial ischemia-reperfusion injury: a neglected therapeutic target. J Clin Invest 2013;123:92100.

7. Lou Z, Wu W, Chen R, Xia J, Shi H, Ge H, et al. Microarray analysis reveals a potential role of lncRNA expression in remote ischemic preconditioning in myocardial ischemia-reperfusion injury. Am J Transl Res 2021;13:234-52.

8. Donato M, Buchholz B, Rodríguez M, Pérez V, Inserte J, García-Dorado D, et al. Role of the parasympathetic nervous system in cardioprotection by remote hindlimb ischaemic preconditioning. Exp Physiol 2013;98:425-34.

9. Weinreuter M, Kreusser MM, Beckendorf J, Schreiter FC, Leuschner F, Lehmann LH, et al. CaM Kinase II mediates maladaptive post-infarct remodeling and pro-inflammatory chemoattractant signaling but not acute myocardial ischemia/ reperfusion injury. EMBO Mol Med 2014;6:1231-45.

10. Liu X, Chen H, Yan Z, Du L, Huang D, Gao WD, et al. Remote liver ischemic preconditioning attenuates myocardial ischemia/ reperfusion injury in streptozotocin-induced diabetic rats. Sci Rep 2021;11:1903. 
11. Zhang J, Yu P, Hua F, Hu Y, Xiao F, Liu Q, et al. Sevoflurane postconditioning reduces myocardial ischemia reperfusion injury-induced necroptosis by up-regulation of OGT-mediated O-GlcNAcylated RIPK3. Aging (Albany NY) 2020;12:25452-68.

12. Hashmi S, Al-Salam S. Acute myocardial infarction and myocardial ischemia-reperfusion injury: a comparison. Int J Clin Exp Pathol 2015;8:8786-96.

13. Zhang J, Zhu L, Li H, Tang Q. Electroacupuncture pretreatment as a novel avenue to protect heart against ischemia and reperfusion injury. Evid Based Complement Alternat Med 2020; 2020:9786482.

14. Yang Q, Mao H, Chen X, Zhang Y, Zhang X, Liu Z, et al. Neiguan (PC6)-based acupuncture pretreatment for myocardial ischemia reperfusion injury: a protocol for preclinical systematic review and meta-analysis. Medicine (Baltimore) 2020;99: e20792.

15. Ni X, Xie Y, Wang Q, Zhong H, Chen M, Wang F, et al. Cardioprotective effect of transcutaneous electric acupoint stimulation in the pediatric cardiac patients: a randomized controlled clinical trial. Paediatr Anaesth 2012;22:805-11.

16. Wang Q, Liang D, Wang F, Li W, Han Y, Zhang W, et al. Efficacy of electroacupuncture pretreatment for myocardial injury in patients undergoing percutaneous coronary intervention: a randomized clinical trial with a 2-year follow-up. Int J Cardiol 2015;194:28-35.

17. Cai RL, Hu L, Zhou YP, Wu ZJ, Wang KM, Tang XM, et al. [Effects of electroacupuncture of "Shenmen" (HT 7) and "Zhizheng" (SI 7) on cardiac function and electrical activities of cardiac sympathetic nerve in acute myocardial ischemia rabbits]. Zhen Ci Yan Jiu 2007;32:243-6. Chinese.

18. Cai RL, Shen GM, Wang H, Guan YY. Brain functional connectivity network studies of acupuncture: a systematic review on resting-state fMRI. J Integr Med 2018;16:26-33.

19. Cai RL, Cui S, Wu ZJ, He L, Yu Q, Wang J, et al. [Effect of electroacupuncture at "Shenmen" (HT 7)- "Tongli" (HT 5) of heart meridian on neuronal activities in paraventricular nucleus of hypothalamus in myocardial ischemia rats]. Zhen Ci Yan Jiu 2018;43:406-13. Chinese.

20. Shao XF. Study on the mechanism of cerebellar fastigial nucleuslateral hypothalamic area in acupuncture preconditioning improving acute myocardial ischemia-reperfusion injury [master's thesis]. Hefei: Anhui University of Chinese Medicine; 2019. p. 1-3.

21. Lee JY, Lin R, Nguyen H, Russo E, Liska MG, Lippert T, et al. Central and peripheral secondary cell death processes after transient global ischemia in nonhuman primate cerebellum and heart. Methods Mol Biol 2019;1919:215-25.

22. Onat F, Cavdar S. Cerebellar connections: hypothalamus. Cerebellum 2003;2:263-9.

23. Cao BB, Huang Y, Lu JH, Xu FF, Qiu YH, Peng YP. Cerebellar fastigial nuclear GABAergic projections to the hypothalamus modulate immune function. Brain Behav Immun 2013;27:8090.

24. Fu LW, Longhurst JC. Electroacupuncture modulates vlPAG release of GABA through presynaptic cannabinoid CB1 receptors. J Appl Physiol (1985) 2009;106:1800-9.

25. Guan F, Ma SL, Chen BY. [Effects of electro-acupuncture on signal transduction pathway of hypothalamic neuroendocrine system in ovariectomized rats]. Zhong Xi Yi Jie He Xue Bao 2009;7:563-8. Chinese.

26. Hao X, Liang J, Zeng T, Sun J, Yuan J, Wu J, et al. [Effects of twisting for reinforcing and reducing methods in acupuncture on GABA and Glu in cerebella of spontaneously hypertensive rats]. J Emerg Tradit Chin Med 2020;29:584-8. Chinese.

27. Wu S, Cao J, Zhang T, Zhou Y, Wang K, Zhu G, et al. Electroacupuncture ameliorates the coronary occlusion related tachycardia and hypotension in acute rat myocardial ischemia model: potential role of hippocampus. Evid Based Complement Alternat Med 2015;2015:925987.

28. Yang TR, Miao YB, Zhang RP, Yu JW. [Establishment of an ex vivo myocardial ischemia-reperfusion model in tree shrews]. Nan Fang Yi Ke Da Xue Xue Bao 2018;38:205-10. Chinese.

29. Khazipov R, Zaynutdinova D, Ogievetsky E, Valeeva G, Mitrukhina O, Manent JB, et al. Atlas of the postnatal rat brain in stereotaxic coordinates. Front Neuroanat 2015;9:161.

30. Curtis MJ, Walker MJ. Quantification of arrhythmias using scoring systems: an examination of seven scores in an in vivo model of regional myocardial ischaemia. Cardiovasc Res 1988; 22:656-65.

31. Zhou L, Zang G, Zhang G, Wang H, Zhang X, Johnston N, et al. MicroRNA and mRNA signatures in ischemia reperfusion injury in heart transplantation. PLoS One 2013;8:e79805. Erratum in: PLoS One 2014;9:e101640.

32. Han Y, Chen S, Wang H, Peng XM. Electroacupuncture pretreatment regulates apoptosis of myocardial ischemiareperfusion injury in rats through RhoA/p38MAPK pathway mediated by miR-133a-5p. Evid Based Complement Alternat Med 2021;2021:8827891.

33. Xu JJ, Zhang L, Ge W, Yong Y, Xie CL, Feng JJ, et al. [Effect of electroacupuncture pretreatment on differential expression profiles of myocardial long non-coding RNA and mRNA in mice with myocardial ischemia-reperfusion injury]. Zhen Ci Yan Jiu 2020;45:389-95. Chinese.

34. Zhang QZ, Xue AY, Wei W, Pang AM, Cao LN, Liu F. Deletion of $\mathrm{P} 110 \delta$ promotes the development of myocarditis in ApoE-deficient mice by increasing mononuclear cell peritoneal infiltration. Mol Med Rep 2020;22:3629-34.

35. Dai QF, Gao JH, Xin JJ, Liu Q, Jing XH, Yu XC. The role of adenosine $\mathrm{A} 2 \mathrm{~b}$ receptor in mediating the cardioprotection of electroacupuncture pretreatment via influencing $\mathrm{Ca}^{2+}$ key regulators. Evid Based Complement Alternat Med 2019;2019: 6721286. 
36. Zhao L, Li D, Zheng H, Chang X, Cui J, Wang R, et al. Acupuncture as adjunctive therapy for chronic stable angina: a randomized clinical trial. JAMA Intern Med 2019;179:1388-97.

37. Shao M, Li Y, Cui H, Jiang M, Tan Q. [Protective effect of acupuncture preconditioning on oxidative stress injury induced by myocardial ischemia-reperfusion injury in rats]. Zhongguo Zhen Jiu 2017;37:285-90. Chinese.

38. Dergacheva O, Yamanaka A, Schwartz AR, Polotsky VY, Mendelowitz D. Direct projections from hypothalamic orexin neurons to brainstem cardiac vagal neurons. Neuroscience 2016;339:47-53.

39. Zhang C, Luo W, Zhou P, Sun T. Microinjection of acetylcholine into cerebellar fastigial nucleus induces blood depressor response in anesthetized rats. Neurosci Lett 2016;629:79-84.

40. Wang M, Guo J, Dong LN, Wang JP. Cerebellar fastigial nucleus stimulation in a chronic unpredictable mild stress rat model reduces post-stroke depression by suppressing brain inflammation via the microRNA-29c/TNFRSF1A signaling pathway. Med Sci Monit 2019;25:5594-605.

41. Abulaiti A, Hu D, Zhu D, Zhang R. Influence of fastigial nucleus stimulation on heart rate variability of surgically induced myocardial infarction rats: fastigial nucleus stimulation and autonomous nerve activity. Heart Vessels 2011;26:654-62.

42. Cao BB, Huang Y, Jiang YY, Qiu YH, Peng YP. Cerebellar fastigial nuclear glutamatergic neurons regulate immune function via hypothalamic and sympathetic pathways. J Neuroimmune Pharmacol 2015;10:162-78.

43. Li Y, Zhang X, Chen L, Yang B, Sui R. Cerebellar fastigial nucleus is involved in post-stroke depression through direct cerebellar-hypothalamic GABAergic and glutamatergic projections. Exp Ther Med 2019;18:2885-92.

44. Gao L, Zhao H, Zhu T, Liu Y, Hu L, Liu Z, et al. The regulatory effects of lateral hypothalamus area $\mathrm{GABA}_{\mathrm{B}}$ receptor on gastric ischemia-reperfusion injury in rats. Front Physiol 2017;8:722.

45. Lu MJ, Yu Z, He Y, Yin Y, Xu B. Electroacupuncture at ST36 modulates gastric motility via vagovagal and sympathetic reflexes in rats. World J Gastroenterol 2019;25:2315-26.

46. Lu M, Chen C, Li W, Yu Z, Xu B. EA at PC6 promotes gastric motility: role of brainstem vagovagal neurocircuits. Evid Based Complement Alternat Med 2019;2019:7457485.

47. Xiao LY, Wang XR, Yang Y, Yang JW, Cao Y, Ma SM, et al. Applications of acupuncture therapy in modulating plasticity of central nervous system. Neuromodulation 2018;21:762-76.

48. Cheung DG, Aizenberg D, Gorbunov V, Hafeez K, Chen CW, Zhang J. Efficacy and safety of sacubitril/valsartan in patients with essential hypertension uncontrolled by olmesartan: a randomized, double-blind, 8-week study. J Clin Hypertens (Greenwich) 2018;20:150-8. 